\title{
TOTAL HIP REPLACEMENTS AT KIKUYU HOSPITAL, KENYA
}

J. Kingori, MBChB, MMed (Ortho), Consultant Orthopaedic Surgeon, PCEA Kikuyu Hospital and L.N. Gakuu, MBChB, MMed (Surg), FCS (ECSA), Associate Professor, Department of Orthopaedic Surgery, College of Health Sciences, University of Nairobi, P.O. Box 19676 - 00202, Nairobi, Kenya

Correspondence to:Dr.J.Kingori, PCEA Kikuyu Hospital, P.O.Box 1010 Kikuyu, Kenya.Email:Johnkingori@gmail.com

\section{ABSTRACT}

Background: Total joint arthroplasty is a highly effective procedure that is frequently performed in elderly patients. This is not so in the third world and is not frequently performed. Total hip replacement (THR) relieves the pain and functional disability experienced by patients with moderate to severe arthritis of the hip, improving their quality of life. It is a highly cost-effective procedure. Many patients deserving this operation cannot afford it in this part of the world and hence do not have it done.

Objective: To analyse total hip replacement surgical procedures done in a mission orthopaedic hospital in Kenya with emphasis on early complications.

Design: A retrospective hospital based study

Setting: Kikuyu hospital

Methods: Medical records of patients who underwent primary total hip arthroplasty between June 2006 and January 2008 in a sample of 97 patients done surgery at Kikuyu hospital. The patient's medical records were looked at from the time a patient is first seen at the clinic by an orthopaedic surgeon and is recommended for a total hip arthroplasty and is followed up until 6 months after the operation. The difficulties encountered either by the surgeon or the patient during this period were recorded and analysed.

Results: Of the 97 patients seen 99 operations were done and there were, two dislocations, two superficial and one deep wound infections, one upper gastro intestinal bleeding, two Deep Vein Thrombosis, one sciatic nerve neuropraxia, one haematoma formation and one intraoperative femoral fracture. Out of the $\mathbf{9 7}$ patients $\mathbf{4 0}$ of them had the surgery performed more than six months after a proper diagnosis was made and hip arthroplasty recommended. This is mainly due to lack of finances. At operation two cases were really difficult and took longer than the usual timing.

Conclusion: Total hip arthroplasty is a safe operation even in the third world with satisfactory results. Just like in any other surgical procedure difficulties and complications are bound to occur.

\section{INTRODUCTION}

Total hip arthroplasty has been done in Kenya for many years (1). There are only a few medical institutions that are in a position to perform this demanding procedure. The main reasons include: lack of adequate equipments, few trained personnel and the high cost of implants. It is mainly in the teaching and referral hospitals and only a few mission hospitals where these arthroplasty is done. There are quite a number of them though that are performed in the well established private hospitals.

Patients suffering from osteoarthritis are known to visit several medical institutions before they get to understand that surgery can relieve the pain they go through. In addition to the related pain and discomfort, hip OA has substantial economic consequences (2). Most of these patients spend a lot of money on pain relieving medications not to mention the side effects of non steroidal anti-inflammatory drugs. They are not able to carry out their daily activities.
Surgeons get to a dilemma on what to do with young patients who either have hip osteoarthritis or sustain a fracture of the neck of femur followed by avascular necrosis of the femoral head. Some opt to do hip fusions as a salvage procedure since it is cheaper and more or less permanent for the arthritic joints. The non cemented hip prosthesis has been introduced in the Kenyan market in the last one year. This will help in solving some of these problems but unfortunately the cost is prohibitive to many.

\section{MATERIALS AND METHODS}

Between June 2006 and January 2008, 97 patients were done Total Hip Replacement Arthroplasty (THRA) surgery at Kikuyu hospital due to osteoarthritis, rheumatoid arthritis or fracture neck of femur. These patients were first seen at the outpatient clinic. After a diagnosis was made, they were then investigated to ensure that they were fit for surgery. Routinely, the following are done: Full blood count, urea and electrolyte levels, a chest X-ray, random blood sugar. 
Electrocardiogram is not done routinely unless justified from the patient's history or on physical examination.

The patient is admitted a day prior to the surgery. Two pints of previously auto donated blood are available.The skin is prepared using betadine which is painted the night before and early in the morning.

All the surgeries are done under spinal anaesthesia. Intravenous prophylactic antibiotic is given during the administration of the anaesthesia. The patient is put in a lateral position. The skin is prepared again using betadine. In over $95 \%$ of the cases an anterolateral approach is used to expose the hip which is then dislocated. Reaming of both the femur and the acetabulum is done. Cemented implants are then put and the hip is confirmed to be stable. In the remaining cases a posterior approach was used.

Closure is done in layers after irrigation with Normal saline. A drain is not routinely used unless there is excessive bleeding. In this study only two drains were inserted. After recovery, the patient is taken to the ward with an abduction splint in place. This is kept for the first 24 hours. Intravenous antibiotics are given for another three days. Prophylactic subcutaneous low molecular weight heparin is given daily after surgery until the patient leaves the hospital.

A check X-ray is taken immediately after surgery to confirm that the implants are in place. Physical therapy is started after 24 hours. The patient is encouraged to do as much as he can. They ambulate putting the full weight on the operated limb unless otherwise stated by the surgeon.The physical therapists teach the patients on the precautions to take at home. Emphasis is put on avoidance of The patient is allowed home between the fifth and seventh day after surgery depending on how independent he is with transfers and ambulation. The patients go home with two crutches. Routinely patients are next seen at the clinic six weeks later. The wound is inspected to confirm that there are no features of infection and another $\mathrm{X}$ - ray is taken to check on the implants. If the patient has no problem and is walking well they are allowed to use one crutch. After this the patient is followed up for the next one year on a three monthly basis. Another X-ray is taken after one year.

We reviewed the medical records of patients who underwent primary total hip arthroplasty between June 2006 and January 2008 in a sample of 97 patients done surgery at Kikuyu hospital. The patient's medical records were looked at from the time a patient presented at the clinic by an orthopaedic surgeon and is recommended for a total hip arthroplasty and is followed up until six months after the operation. The difficulties encountered either by the surgeon or the patient during this period were recorded and analysed.

\section{RESULTS}

Financial difficulties: Out of the 97 patients 40 of them had the surgery performed more than six months after a proper diagnosis was made and hip arthroplasty recommended. This is mainly due to lack of finances. The majority of our patients do not have a health insurance cover despite the fact that there are a number of insurance companies in Kenya offering these services. They pay for their medical care from their pockets. THRA is mainly done in the elderly patients and it is usually the duty of the patient's sons and daughters to raise the funds for the surgery to be done. This is commonly done through fundraising whereby relatives, neighbours and friends are called upon to give what they can. There are however few of the patients who have an insurance cover or have saved and are able to pay for themselves.

Some patients get lost to follow up in most cases after the first postoperative visit. They only show up incase a problem develops. In this study 35 (34\%) patients got lost to the follow up before six months were over. It is mainly due to financial constraints that this happens despite the fact that the patients know the importance of follow up.

Dislocations: There were two (2.06\%) dislocations post operatively. In both cases a posterior approach had been used. The first one occurred two weeks after the surgery while the second happened after five weeks. Closed reduction in both cases was attempted under spinal anaesthesia but failed. Open reduction had to be done. In the first case the cup was noted to have been over abducted and had to be removed and replaced with another. In the second case the implants were not adjusted. The soft tissues were tightened after reduction using sutures. In the later case, the patient was mentally unstable and was unable to follow instructions. Both hips remained stable after the repeat surgery.

Infections: There were two (2.06\%) wounds with superficial and one (1.03\%) deep infections. The superficial ones were noticed while the patients were still in the ward after surgery before they had been discharged. Patients had no systemic symptoms. In both cases Intravenous antibiotics were given for a week after a culture and sensitivity was done. The patients were discharged after the infection settled on oral antibiotics for another two weeks. In both cases Staphylococci bacteria were grown.

The patient who got the deep infection was diabetic. His blood sugar was not well controlled. He was not a regular diabetic clinic attendant. After the first surgery we did not see him until after three months. He was sickly with pus draining from the incision site. 
His blood sugar was $23 \mathrm{mmol} / \mathrm{L}$. His haemoglobin level was $9 \mathrm{~g} / \mathrm{dl}$. He was initially stabilized and was taken back for surgery. All the implants were removed and he was left with a girdlestone.

Femur shaft fracture: This occurred on a 90 year old lady who had severe osteoarthritis with protrusion of the acetabulum. Her bones were very porotic. Dislocation of the hip was difficult. It was during this stage that one of the assistants rotated the limb and the bone gave way resulting in a spiral midshaft femur fracture. The femoral neck was osteotomised and the head was removed using a cockscrew. The femoral shaft was then plated and then the implants to replace the hip were inserted.

Duration of surgery: On average, a primary THRA surgery takes between 1 to 1.5 hours in this Hospital. There were two cases that took a longer time. The first one is the one mentioned above which lasted for 3.5 hours.

The second case was of a 66 year old man. He had sustained a fracture of his left femoral neck four years before and an Austin moore hemiarthroplasty had been put. He complained of pain with erosion of the acetabulum on the X-ray. We were converting this to a total hip replacement. There was a lot of scar formation and a significant amount of bleeding during the operation.

The most difficult part was removal of the Austin Moore prothesis which had already had soft tissues grow across its fenestrations. We had to use a significant amount of force to hummer it out. This was done in turns. The operation lasted about three hours.

Upper gastro intestinal bleeding: A lady aged 61 years was admitted and done THRA surgery. In her history we had noted that she had used Non steroidal anti inflammatory drugs for more than 2 years. She had experienced some epigastric discomfort on and off but only mildly. She was on omeprazole and antacid syrup. The surgical procedure was uneventful. Her recovery was good till the third post operative day. She woke up well in the morning. It is after she took her breakfast that she started vomiting. Among other things she did vomit frank blood that would amount to 1.5 litres. Her blood pressure was no longer recordable and her pulse was very weak. She got restless. Blood transfusion was started and she was then transferred to another hospital for intensive care. She did recover and was treated for duodenal ulcers.

Haematoma: There was one patient who developed quite a massive haematoma that warranted his being taken back to theatre for it to be evacuated. This was after a normal THRA procedure. It was noted on the second postoperative day. At the second operation, there was no active bleeding noted. The haematoma was evacuated and the patient covered with intravenous antibiotics. Fortunately the patient did not develop any infection.

Deep venous thrombosis: This occurred in two patients, both female aged 58 and 67 years. Both were active even before the surgery. They both developed pain and tenderness on the calf muscles on the fourth and fifth postoperative days respectively. Doppler ultrasound scans done demonstrated venous thrombus on the legs but no thrombi was demonstrated on the thigh and pelvic areas. Both patients were given anticoagulants for three months. They continued being active. They pain disappeared and has not recurred.

Sciatic nerve neuropraxia: This happened in a 48 year old lady who had a coxa vara on her left hip. Her left lower limb was 2 centimetres shorter than the right before the surgery. After the surgery she did gain one and a half centimeters on the affected limb. She had a neuropraxia of her left sciatic nerve with the motor function more affected than the sensory a day after. There was a foot drop. This was observed and the patient reassured. It had recovered after 6 weeks when she came back for review.

This is the initial x-ray of the patient described above. There is a coxa-vara deformity on her left hip.

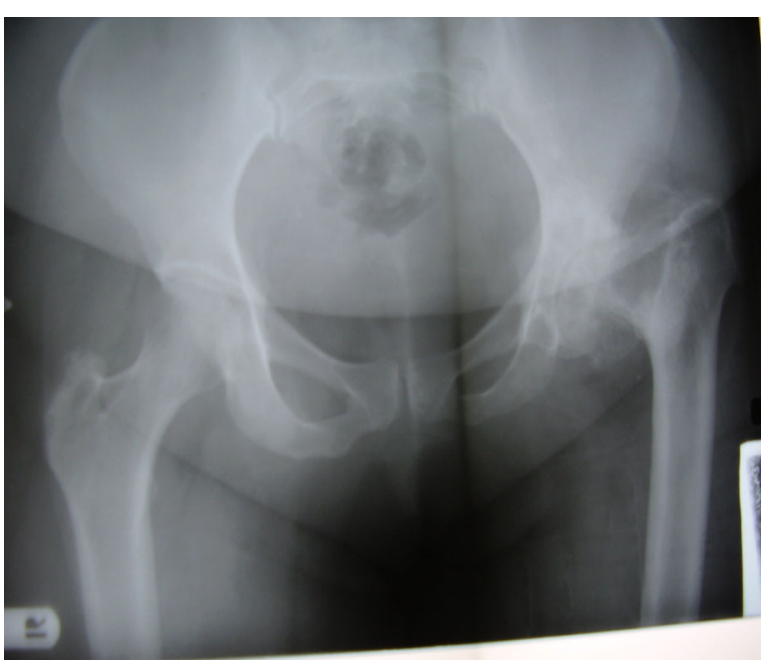

\begin{tabular}{lc} 
Type of difficulty/complication & Number of cases \\
\hline Hip dislocation & 2 \\
Infections & 3 \\
Upper GIT bleeding & 1 \\
Deep venous thrombosis & 2 \\
Sciatic nerve neuropraxia & 1 \\
Haematoma formation & 1 \\
Long duration of surgery(>11/2 hours) & 2 \\
Intraoperative femoral shaft fracture & 2
\end{tabular}




\section{DISCUSSION}

Total hip and knee arthroplasty is the definitive treatment for relieving pain and restoring function in people with moderate to severe osteoarthritis for whom medical therapy has failed (3). However, patients needing elective total hip arthroplasty in publicly funded health care systems often wait because resources do not match demand (4-10):

This essential procedure is not accessible to many patients suffering from this condition in the third world. As stated earlier it is very expensive. Secondly it is only a few medical institutions that have the necessary equipment. Thirdly there is a limited number of surgeons who are able to carry out this procedure successfully. This calls for government policies to ensure that the mentioned areas are taken care of in terms of resource allocation and personnel training.

In terms of the difficulties encountered in this study it is apparent that other surgeons even in the developed world essentially go through the same. In a series of 300 total hip replacements, Lewinnek et al reported a $3 \%$ dislocation rate (11). There were two $(2.06 \%)$ cases of dislocation reported in this study.

The use of perioperative antimicrobial prophylaxis and a laminar airflow surgical environment has reduced the risk of intraoperative infection to less than 1\% after hip and shoulder replacement and to less than $2 \%$ after knee replacement $(12,13)$. Laminar airflow is not available in our set up. Theatres are well aerated though. Prophylactic antibiotics are routinely given during anaesthetic administration. In this study, there were two (2.06\%) wounds with superficial and one $(1.03 \%)$ deep infections.

\section{CONCLUSION}

From the results given above, it is apparent that total hip arthroplasty is a safe operation even in the third world with satisfactory results. Governments need to allocate more resources to this area of orthopaedic surgery in terms of personnel training and patient care to save many patients that live in pain for many years.

\section{REFERENCES}

1. Mulimba, J.A.O. Total hip arthroplasty experience in a developing African country. East Afr. Ortho. J. 2007; 1: 68-73.

2. Hadler, N.M. Osteoarthritis as a public health problem. Clin. Rheum.Dis. 1985; 11: 175-185.

3. Hawker, G.A.,Wright, J.G., Coyte, P.C., et al. Health-related quality of life after knee replacement surgery. J. Bone Joint Surg. Am. 1998; 80: 163-173.

4. Williams, J.I., Llewellyn-Thomas, H., Arshinoff, R., Young, N. and Naylor, C.D. The burden of waiting for hip and knee replacements in Ontario. J. Eval. Clin. Pract. 1997; 3: 59-68.

5. Hadorn, D.C. and Holmes, A.C.The New Zealand priority criteria project. Part 1: Overview. Brit. Med.J. 1997; 314: 131-134.

6. Smith,T.Waiting times:monitoring the total postreferral wait. Brit.Med.J. 1994; 309: 593-596.

7. Niinimaki T. Increasing demands on orthopedic services. Acta. Orthop. Scand. Suppl. 1991; 241: 42-43.

8. Beverland, D.E., McKee, W.S., Murphy, J.S., Mollan, R.A. and Barron, D.W.Development of the Belfast orthopedic information system. Health Serv. Manage. 1989; 85: 270272.

9. Abyholm, G., Riise, G., Melsom, M.N., Piene, H. and Gulbrandsen, I. The waiting list guarantee and its violation. Characteristics of patients who have been waiting more than five months. Tidsskr Nor Laegeforen. 1997; 117: 366-368.

10. Groothoff,J.W.,Kruijer, S.W.and Post,D.What determines waiting time for cataract surgery, knee arthroscopy and total hip arthroplasty and how satisfied are the patients? Ned. Tijdschr. Geneeskd. 1995; 139: 14891493.

11. Derrett, S., Paul, C. and Morris, J.M. Waiting for elective surgery: effects on health-related quality of life. Int. J. Qual HIth. Care. 1999;11:47-57.

12. G. E. Lewinnek, J.L. Lewis, R. Tarr, C.L. Compere, and J.R. Zimmerman Dislocations after total hip replacement attroplasties. J. Bone and Joint Surgery. 60: Issue 2, 217220.

13. NIH Consensus Development Panel on Total Hip Replacement. NIH consensus conference: total hip replacement. JAMA. 1995; 273: 1950-1956. 